\title{
Improving the Energy Efficiency of Propulsion Systems of the Sea and River Fleets
}

\author{
Alexey Fedorovich Burkov ${ }^{1}$, Victor Viktorovich Mikhanoshin ${ }^{2}$, Wagarshak Radikovich Avetisyan ${ }^{3}$ \\ ${ }^{1}$ Far Eastern Federal University, Sukhanova Street, 8, Vladivostok, 690091, Russia \\ ${ }^{2}$ Maritime state university named after G.I. Nevelskoy, Verkhneportovaya Street, 50a, Vladivostok, 690059, \\ Russia \\ ${ }^{3}$ Pacific Higher Naval School named after S.O. Makarov, Kamsky Lane, 6, Vladivostok, 690062, Russia
}

\begin{abstract}
The article considers the main variants of the main power plants of sea and river vessels operating in maneuvering modes, and analyzes ways to improve their energy efficiency, as well as planning vessels, including hydrofoils. On the basis of the performed researches the hybrid power plant providing the coefficient of loading of the main engine close to nominal values at change of speed of the vessel in a wide range is offered. Also proposed is the structure of the main power plant for hydrofoil ships, which has the best environmental and energy performance compared to known installations.
\end{abstract}

Key words: Hybrid Power Plant, Main Power Plant, Rowing Electric Plant, Sea and River Vessels, Primary Engine, Propulsion System, Energy Efficiency

\section{INTRODUCTION}

A characteristic feature of the operation of small-tonnage vessels of coastal and intraport navigation of the sea and river fleets is the operation of their main power plants (MPP) and propulsion systems (PS) in General with frequent transient and shared modes. The performed analysis allows to draw a conclusion that as the main primary engines (PE) the main PS of such vessels in most cases are used diesel engines. Thus, there is a complex of the problems connected with an increase of low economic efficiency of work of PS (diesels) owing to the fact that the power of such PS has an approximately linear dependence on the frequency of rotation of its shaft, and power on a propeller shaft is proportional to the third power of the frequency of rotation of its shaft.

The method of calculation and selection of PE and propellers for their joint work in the system of propulsion systems (PS) of sea and river vessels in many cases is based on the fact that the developed power of PE at nominal frequencies of rotation of their shafts should be fully balanced by the power consumed by the propellers of ships.

In these cases at any other speeds of rotation of propellers, smaller nominal in an admissible range and characteristic for transition and share modes, there is an excess of the developed PE of the main MPP of capacities as the main diesel PE in such modes work at the lowered coefficients of loadings on the moment, and accordingly, power. In addition, the operation of the main diesel PE in partial fractional and transient modes is characterized by reduced values of their efficiency (efficiency) and increased specific consumption of diesel fuel and lubricants. Thus there is incomplete combustion of fuel mixtures in the combustion chambers of PE, which leads to an increase in carbonization and accelerated coking piston rings of diesel engines and, as a consequence, to a reduction of component life and the main PE in General. These circumstances are the main reason for the increase in the concentration of toxic substances in the exhaust gases of marine and diesel PE.

One of the most effective ways to improve the technical and economic performance in the operation of the main diesel engines is to ensure their stable stationary operation with nominal rotational speeds $n_{e n}$ and nominal $M_{e n}$ moments on the shaft under variable loads in the permissible ranges.

At the present stage of development of marine and river shipbuilding, despite the available scientific and technical studies, the tasks associated with increasing the technical and economic indicators in the operation of marine diesel PE in modes other than nominal, are relevant and require further solutions aimed at eliminating the shortcomings, the main of which are listed above.

\section{THEORETICAL ANALYSIS}

In shipbuilding of the present time there are several main scientific and technical directions, allowing to provide partial solution of certain tasks $[1,2]$. These include:

- application in the composition of PS vessels propellers with rotating blades (adjustable pitch screws (APS));

- installation on vessels of several similar main PE having identical characteristics and working on one common propeller shaft;

- the use of marine PS of different types of main PE (diesels and electromechanical converters (EMC), etc.), working on one common propeller shaft;

- use in the composition of PS rowing electrical installations (REI); 
- application of reversible generator sets in marine PS.

1. When using propellers with rotating blades (APS) in marine PS, as a rule, two control modes are provided: combinatorial mode and control mode at a constant (nominal) speed [3].

In the combinatorial control mode, the change in the speed of the active movement of the vessel is carried out by changing both the rotation frequency of the main PE shaft and the rotation angle of the propeller blades. Combinatorial mode is used mainly when performing maneuvers in different conditions (when changing the speed and trajectory of ships in the port, when passing narrowness of vessels, etc.).

In cases of using the control mode at a constant (nominal) frequency of rotation of the main PE shaft, the speed control of the vessel is carried out by changing the angle of rotation of the propeller blades (propeller pitch). The constant frequency of rotation of the PE shaft gives the basis for connection to it in this mode of auxiliary generators-shaft-generator (SG). But it is not possible to optimize the operation of the ship's PS in different modes.

The ability to set with the help of two independent parameters (speed and pitch of the propeller) the required operating modes in a relatively wide range significantly expands the possibilities of using PS, as changing these parameters can be provided by the main ship PE at any permissible screw, regulatory and restrictive characteristics, allows you to maintain optimal modes when changing external operating conditions.

At constant speed of movement of the vessel with APS in process of increase in a step of the propeller with decrease in frequency of rotation of a propeller shaft the total value of efficiency of PS increases, reaching the greatest value at a constructive angle of a turn of blades of a propeller shaft (APS step) near limiting characteristic.

Thus, the use in the composition of the ship's PS rowing APS allows you to more effectively solve the problem of improving economic performance (efficiency of screws, etc.) in the main MPP.

2. At installation on vessels of several identical main PE having identical characteristics and working on one General propeller shaft (Figure 1) there is a possibility of partial switching-off of PE in share modes of operation of PS. At the same time the load on the remaining main engines increases and can reach nominal values.

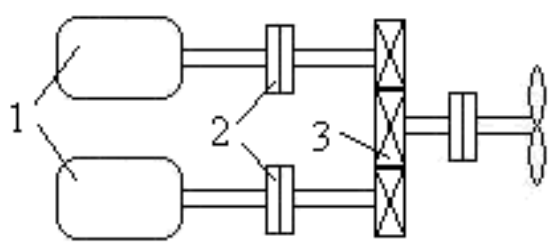

Figure 1: The scheme of the PS of the ship, including several of the same type of home PE have identical characteristics

In Figure 1: 1-the main PE; 2 - disconnecting controlled couplings; 3 -summing (common) reducer.
Partial shutdown of the main PE in the shared modes of operation of the PS is difficult or impossible in cases where the change of operating modes, including starts/stops of the main PE (diesels) and the change of engine loads in wide ranges (from minimum to full speed of the vessel) occurs relatively often, the operation of the main PE in such modes leads to increased wear of their components and the PS as a whole.

In addition, to ensure the nominal load factor of PE remaining in operation, it is necessary to have three or four main engines in the main MPP and an appropriate number of disconnecting clutches. At the same time, the mass-dimensional characteristics of the main MPP increase, their complexity increases and cost indicators increase.

3. The use of different types of main PE, working on one common propeller shaft (Figure 2), eliminates the main drawback inherent in marine PS with several of the same type of main PE, having identical characteristics, which work on one common propeller shaft (Figure 1). In Figure 2: 1-main PE; 2, 4, 5-disconnecting controlled couplings; 3-common reducer; 6-reversible synchronous EMC; 7-semiconductor Converter (SC); 8-power source.

The main characteristic features of the schemes, a variant of which is presented in Figure 2, is the ability to connect reversible synchronous EMC for operation in the modes of rowing electrical engines (EE) with almost any required frequencies.

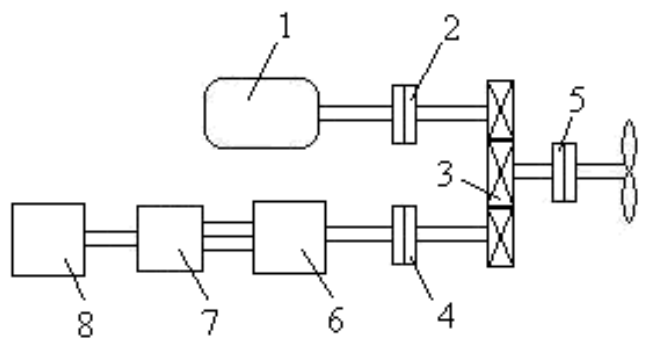

Figure 2: The scheme of ship PS, which includes several main different types of PE

Regulation of the electromagnetic moment of the EMC when operating in the rowing EE mode is carried out by changing the voltage and frequency of the current supplied to its windings by means of the SC. In this case, the sources of electricity (EE) for the connected EMC can serve as auxiliary diesel generators (D-E) or relatively powerful batteries, for example Lithium Iron Phosphate battery modules [4].

In the first case, the D-E will operate in modes other than stationary (with constant shaft speeds and variable values of load moments). Based on the above, such schemes ship PS inherent in almost all the main drawbacks characteristic of the traditional main sea.

In the second case, when batteries are used as sources of electricity, allowing to provide constant moments of static EMC loads in GE modes, marine PS have the best economic, operational and environmental characteristics.

The main drawbacks of the ship's PS scheme, presented in Figure 2 and including several different types of main PE, include the presence of a summing (common) reducer 3 and 
three disconnecting controlled couplings 2, 4 and 5, which reduce the reliability of the main MPP.

\section{HYBRID POWER PLANTS}

The main drawbacks of hybrid schemes of marine PS, including several different types of main PE, a variant of which is shown in Figure 2, can be eliminated by using the main hybrid MPP serial type with buffer accumulator batteries in the DC link - Figure 3 [5]. The AC rectifier UZ1 based on bipolar transistors with isolated gate (IGBT) consumes almost sinusoidal current and has a power factor $(\cos \varphi)$ of about 0.99 , so that the distortion introduced by the rectifier UZ1 into the supply network with a current source in the form of the main D-E (M-G) is minimal.

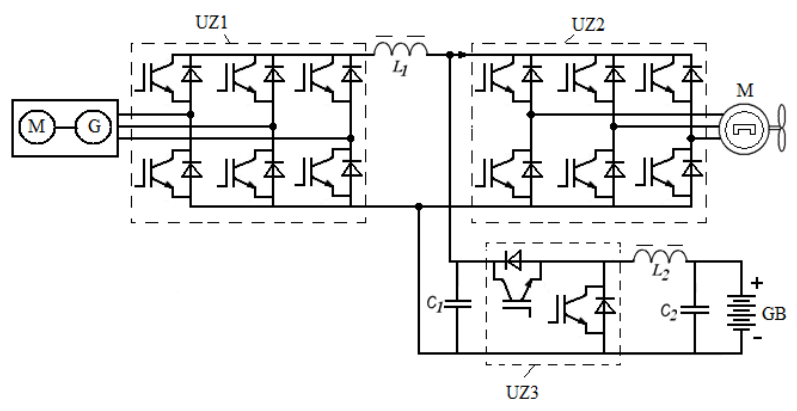

Figure 3: Diagram of a serial hybrid power plant with buffer batteries

Rowing EE M three-phase AC receives power from the Converter UZ2. Changing the speed of the vessel is carried out by changing the frequency of rotation of the propeller shaft by means of frequency regulation of the voltage supplied to the propeller EE. With the help of a two-way pulse Converter UZ3 provides the selection of excess power from the main D-E for charging traction accumulator batteries GB at relatively low speeds of the vessel $[6,7]$. Converter UZ3 is also involved in the implementation of parallel operation of two sources (main D-E with rectifier UZ1 and accumulator batteries), which is used when moving the ship in maneuvering modes, when there is a need for increased power on the propeller.

Synchronous motors with excitation from permanent magnets made of rare earth materials, which have high efficiency values and increased reliability, can be used as rowing EE M (Figure 3) [8]. In comparison with asynchronous EE their $\cos \varphi$ can be numerically equal to one not only in nominal, but also in partial modes of operation. When the ship is decelerated, the propeller EE $\mathrm{M}$ be switched to generator mode, creating a braking electromagnetic moment. The mechanical energy converted into electrical energy during regenerative braking is used to recharge the GB accumulator batteries.

As buffer batteries GB (Figure 3) it is advisable to use a set of lithium-ion cells. Lithium-ion batteries have a relatively high voltage of one cell, have a higher service life, have an increased energy density, are able to quickly charge and give a large current. In particular, lithium-iron-phosphate accumulator batteries (lithium Iron Phosphate, LiFePO4, LFP) in operation are able to provide up to 5000 charge-discharge cycles at $70 \%$ of the discharge capacity, have high efficiency values (up to $94 \%$ ). They have increased operational reliability and have fire safety. Along with the ability to charge quickly, they, in comparison with other types of lithium-ion batteries ( $\mathrm{LiCoO} 2, \mathrm{LiMn} 2 \mathrm{O} 4$, etc.), more slowly lose capacity. The loss of $20 \%$ of the capacity of LiFePO4 batteries is observed after the completion of at least 2000 charge-discharge cycles. Unlike other types of lithium accumulator batteries, lithium-iron-phosphate batteries have an almost constant output voltage, amounting to about $3.2 \mathrm{~V}$ during discharge until its termination, which allows such accumulator batteries to operate at a capacity close to full, until their permissible discharge.

The scheme shown in Figure 3 contains a relatively small number of elements, has increased reliability due to the absence of disconnecting clutches and reducers in it. Such schemes of PS with double conversion of electric power, but without energy storage, are well known and widely used on vessels with electric propulsion, have proved their viability and reliability.

A higher energy efficiency value of the proposed main ship hybrid MPP can be achieved by charging buffer accumulator batteries from the shore network (for example, when the ship is moored at night).

4. When used as part of the ship's PS MPP main PE work in the same way as the auxiliary D-G in the scheme of PS, presented in Figure 2. The main MPP with rowing EE by virtue of their specific features have found distribution on ships for various purposes (icebreakers, ferries, etc.). Along with ship-based PS, including APS, PS-based REI are almost the only possible use for frequent reversals and speed changes in the movement of ships. In comparison with the main MPP, including APS, PS with REI are more reliable, but less economical, having increased weight and size indicators. In addition, the main disadvantages of such PS include the presence of several successive stages of energy conversion: the chemical energy of the fuel into the mechanical energy of the main PE; mechanical energy of the main PE into electrical energy generated by the main generators (MG) REI (direct conversion); electrical energy into mechanical energy rowing EE (reverse conversion). The resulting efficiency of such PS has relatively low values.

5. The use of reversible generator sets in marine PS involves the use of synchronous reversible EMC (SG) hung on the propeller shafts in two main modes of operation: in the generator (at full speed of the vessel) and in the engine (when the vessel is moving in shared modes). Provision of electric power of the ship SG working in motor modes, as well as in the ship PS including several different types of the main PE (Figure 2), it is possible from auxiliary D-G or from accumulator batteries. Since the capacity of the ship's SG is approximately $10 \ldots 15 \%$ of the capacity of the main PE, it is not impossible to significantly increase the energy efficiency of PS vessels through the use of such power plants.

The performed analysis of the main directions of development of the main MPP and options of increase of their power efficiency existing in modern shipbuilding gives the basis to draw a conclusion that till now there are no 
enough effective actions allowing to provide stationary modes of operation of the main PE in the conditions of variable loadings.

It is known that in coastal (land) vehicles, the reduction of dynamic loads on the main PE is carried out through the use of reversible EMC, working together with them in motor modes, of power commensurate with the main PE, receiving electricity from the traction accumulator battery. Solving the problems of using similar hybrid schemes of parallel type, consisting of two different types of comparable power engines in conjunction with rowing APS, in marine PS in order to improve their energy efficiency, refers to the current directions of development of the main MPP [9].

As a basis for the main hybrid MPP within the objectives it seems appropriate to use the scheme presented in Figure 2. At the same time, it is expedient to control the modes of operation of the ship's hybrid PS by simultaneous control actions on the APS and two-way SC. At the moment of resistance on APS $\mathrm{M}_{1}$, less than the nominal value of the moment $M_{e n}$ of the main PE $\left(M_{1}<M_{e n}\right)$, the angle of rotation of the blades of APS will be equal to the value $\alpha_{1}$ - Figure 4 .

The EMC of comparable power in this case operates in the generator mode (SG), creating on the shaft the necessary and sufficient additional mechanical braking torque $\Delta M$, the value of which is equal to the difference between the nominal torque $M_{e n}$ main PE and the actual moment of resistance $M_{p}$ APS, i.e.

$$
\Delta M=M_{e n}-M_{p} .
$$

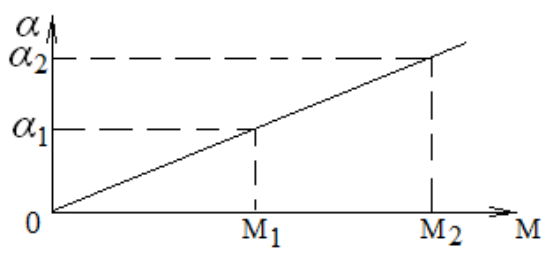

Figure 4: Graph of the dependence of the rotation angle of the APS blades on the moment of the main PE in the hybrid scheme of the parallel type PS

The EMC of alternating current electricity generated by the EMC operating in the generator mode is used to power the ship's electricity consumers or after preliminary rectification of the SC to recharge the traction accumulator batteries.

If it is necessary to increase the speed of the vessel increases the angle of rotation of the blades APS. In this case, the EMC by means of a remote automated control system is transferred to the motor mode, providing the SC frequency control of the shaft revolutions. When the EMC operates in the motor mode, the traction accumulator battery serves as a source of electricity for its power supply. To achieve the maximum speed of the vessel, the angle of rotation of the APS blades is increased to the value $\alpha_{2}$ corresponding to the moment $M_{2}\left(M_{2}=M_{e n}\right)$ - Figure 4.

When the voltage and/or capacity of traction accumulator batteries are reduced to the minimum permissible values, their further discharge stops and, as there is excess power of the main PE (at values of the rotation angles of the blades of the APS, less than $\alpha_{2}$ ), the charging process is performed. In General, with such a scientific and technical solution, the traction battery in the hybrid scheme is used in the buffer mode, giving electricity when the main PE power is insufficient, and consuming electricity in cases of excess power on the main PE shafts.

Thus, at the given layout of the scheme of the main ship MPP there is an opportunity to minimize the shortcomings peculiar to PS known in shipbuilding of the present time. The proposed hybrid scheme has relatively high efficiency values, since the transmission of torques from the main PE to the propellers is carried out directly. In addition, in such a hybrid scheme, the main PE of lower power can be used, based on the average values of the power on the shafts. The increased power values required in dynamic modes, for example during acceleration of ships, are provided by EMC operating in the propulsion mode, which at the same time receive electricity from traction accumulator batteries. As a result, the main PE in operation in the hybrid schemes under consideration, regardless of the speed of the vessels, operate in modes close to the nominal ones. At the same time, along with the increase in operating resources of the main PE, lower specific fuel and lubricant consumption, the environmental characteristics of hybrid PS are improved, due to the minimum volumes of emission of harmful substances into the surrounding atmosphere.

The use of such hybrid schemes allows to improve the energy efficiency of the main MPP and high-speed vessels. It is known that in order to accelerate a high-speed (planing) vessel and get it out of the displacement mode of movement into the hydrofoil mode, i.e. the planing mode, and overcome the wave crisis arising in this case, it needs two or three times the power from the steady-state mode of movement in this high-speed mode. Thus, on high-speed vessels, it is necessary to install sufficiently powerful main PE to ensure such relatively short-term operation modes. After overcoming the ship's hull wave crisis and its output in the planing mode of movement on the propeller shaft requires much less power. It is obvious that in this long-term operation mode, the main PE operate with low load factors, which limits the resource of their work, increases the specific consumption of fuel consumed by them, increases the content of harmful substances in the exhaust gases. Thus, for such high-speed planing vessels, the task of providing nominal load factors of their main PE in characteristic long-term operating modes with sufficient reserves of the main MPP capacities necessary for short-term operating modes arising when the vessels enter the planing modes is urgent.

An option for solving this urgent scientific and technical problem can be the installation on ships of additional accelerator engines in the form of synchronous reversible EMF 6, connected to the summing (common) gearboxes 3 propeller shafts by means of disconnecting couplings $4-$ Figure 2. When working in motor mode EMF 6 is connected to an additional source of electricity 8 in the form of accumulator batteries through a reversible SC 7 .

Acceleration of the vessel in this case is made at the closed disconnecting couplings 2,4 and 5 with the propeller drive simultaneously from the main PE 1 and from the synchronous EMC 6 working thus in the motive mode. After overcoming the ship's hull wave crisis and its output in the 
planning mode, the disconnecting coupling 4 is opened and the power supply of the EMC 6 is disconnected. The propellers are rotated from this moment only by the main PE 1.

Additional advantages of high-speed vessels with the main hybrid sea are due to the possibility of their movements with relatively low speeds, which are necessary, for example, at the entrance (exit) of vessels in the waters of ports, only due to the operation of EMC 6 in motor modes. At the same time, the operational resource of idle main PE is saved and there is practically no pollution of the environment by exhaust gases, the limit values of which are set in [10].

In addition, in cases of short-term Parking of passenger high-speed vessels at the berths (during disembarkation boarding of passengers, etc.) with hybrid main MPP, it is possible to operate the main PE 1 (Figure 2) at nominal static moments, excluding uneconomical operation of the PS at idle. In these modes of operation, the disconnecting coupling 5 is in the open state, and the EMC 6 is transferred to the generator mode, providing through the reversible SC 7, operating in the rectifier mode, charging the accumulator batteries 8 .

Calculation and selection of traction batteries for hybrid main MPP is carried out on the basis of the following conditions:

$$
P_{c}=U_{c} I_{c} ; I_{c}=k_{n} C_{b} \text {. }
$$

In relations (2) $P_{c}$ - charging power of traction battery, Wt; $U_{c}$ - average charging voltage, $\mathrm{V} ; I_{c}$ - the charging current, $\mathrm{A} ; k_{n}$ - coefficient depending on the type of traction battery used, $1 / \mathrm{h}\left(k_{n}=0,1 \ldots 0,3\right) ; C_{b^{-}}$the capacity of the traction battery marine PS, A.h, commensurate with the rated power of the main $\mathrm{PE}$ [4].

\section{CONCLUSI4ON}

Thus, the performed researches give the basis to draw a conclusion about expediency of the scientific and technical researches directed development and introduction for low-tonnage vessels of hybrid main MPP.

\section{REFERENCES}

[1] G. A. Konakov, B. V. Vasiliev. Ship power plants and technical operation of the fleet. Under the General editorship of G. A. Konakovo, Transport, Moscow, 1980.

[2] I. P. Fiyas, V. V. Evgrafov. Reversible halogen generator set with semiconductor frequency Converter: collection of scientific works, V/O marine technical information reclami, Moscow, 1987, 68-74.

[3] G. A. Popov. Ship diesel control Systems, GMA publishing house named after Admiral S. O. Makarov, Saint-Petersburg, 2008.

[4] V. Bruns. E-mobility solutions, Journal Electric \& Hybrid Vehicle Technology International, January 2014. Available from: http://viewer.zmags.com/publication/70ad7366\#/70ad $7366 / 1$

[5] R. Strzelecki, G. Benysek. Power Electronics in Smart Electrical Energy Networks, Springer, 2008. https://doi.org/10.1007/978-1-84800-318-7

[6] N. Mohan, T. M. Unndeland, W. P. Robbins. Power Electronics: Converters, Applications, and Design, John Wiley \& Sons, Inc., 1995.

[7] M. H. Rashid. Power Electronics Handbook, Elsevier, Inc., 2007.

[8] J. Gieras, M. Wing. Permanent magnet motor technology: design and applications, Marcel Dekker, Inc., 2002.

[9] RF patent No. 2483972 Method of control of ship combined power plant; publication 10.06.2013.

[10] The International Convention for the Prevention of Pollution from Ships, 1973 as modified by the Protocol of 1978 (MARPOL 73/78, MARPOL is short for maritime pollution and 73/78 short for the years 1973 and 1978). 